\title{
Aktueller ZEBS Warencode für Feinkosterzeugnisse
}

\author{
M. Busch \\ Landesuntersuchungsanstalt für Gesundheits- und Veterinärwesen des Freistaates Sachsen
}

\section{Zusammenfassung/Erläuterung}

Die Unterteilung des Warencodes 20 beinhaltet seit der letzten Überarbeitung auch alle obst- und gemüsehaltigen Salate. Eine weitere Unterteilung erfolgt nach Präserve, Konserve und brennwertreduziert. Daraus ergeben sich je nach Art des Feinkostsalates unterschiedliche mikrobiologische Untersuchungsumfänge. Die von der DGHM empfohlenen Richt- und Warnwerte für Feinkostsalate (Entwurf einer Aktualisierung durch die Arbeitsgruppe „Mikrobiologische Richt- und Warnwerte“ der Fachgruppe „Lebensmittelmikrobiologie und -hygiene“ der DGHM und durch die Arbeitsgruppe „Lebensmittelmikrobiologie“ der SVUA/CVUA NRW vom 09.06.05) sind nur bedingt anwendbar.
Für relativ unbehandelte gemüse- bzw. obsthaltige Salate sind die Werte zu streng und die Parameter nur bedingt geeignet. Für Konserven sind die angegebenen Werte ebenfalls nicht anwendbar, da diese steril sein sollten.

\section{Diskussionsergebnis}

Herr Dr. Häger (SVUA Arnsberg) wird beauftragt, die dargestellte Problematik - mit der Bitte um Klärung des Sachverhalts - an die DGHM heranzutragen. 